\section{Safety and efficacy of manual small incision cataract surgery for brunescent and black cataracts}

R Venkatesh', CSH Tan², GP Singh', K Veena', KT Krishnan ${ }^{1}$ and RD Ravindran ${ }^{1}$

\section{Abstract}

Aims To evaluate the safety, visual outcome and complications of manual small incision cataract surgery (MSICS) in the treatment of patients with brunescent and black cataract (BBC).

Methods In a non-randomised interventional case series, 102 consecutive patients with $B B C$ underwent cataract extraction by MSICS, with staining of the anterior capsule by trypan blue.

Results Of the 102 eyes with BBC, MSICS was performed through superior scleral tunnel (SST) in 31 eyes (30.4\%) and through temporal scleral tunnel (TST) in 71 eyes $(69.6 \%)$. The main intraoperative complication was posterior capsule rupture in two patients $(2.0 \%)$. Postoperatively, 20 eyes (19.6\%) developed corneal oedema. Mild iritis was seen in six eyes $(5.9 \%)$ and moderate iritis with fibrin membrane formation occurred in three eyes $(2.9 \%)$. On the 40th postoperative day, 80 patients (78.4\%) achieved uncorrected visual acuity of $6 / 18$ or better, and $99(97.1 \%)$ had best-corrected visual acuity of $6 / 18$ or better. Patients in the SST group had significantly higher postoperative astigmatism compared to those in the TST group ( $-1.08 \mathrm{D}$ vs $-0.72 \mathrm{D}, P=0.017)$. Conclusion MSICS with trypan blue staining of the anterior capsule is a safe and effective method of cataract extraction for patients with BBC.

Eye (2009) 23, 1155-1157; doi:10.1038/eye.2008.190; published online 20 June 2008

Keywords: brunescent and black cataract; manual small incision cataract surgery; visual acuity

\section{Introduction}

Manual small incision cataract surgery (MSICS) is an inexpensive alternative to phacoemulsification and achieves better uncorrected visual acuity (UCVA) compared with extra capsular cataract extraction (ECCE). 1,2 We report the safety and efficacy of MSICS in brunescent and black cataracts (BBCs).

\section{Patients and methods}

This prospective study included 102 eyes of 102 consecutive patients with $B B C$ who had routine MSICS operations at Aravind Eye Hospital, Pondicherry, India, between January 2006 and May 2006. Cataracts appearing red-brown on slit-lamp examination were classified as BBCs were distinguished by the colour. ${ }^{3}$ Patients with small pupils $(<5 \mathrm{~mm})$ due to pseudoexfoliation or diabetes were excluded. Preoperative examination included slit-lamp examination, tonometry, keratometry, and biometry.

\section{Surgical technique}

Retrobulbar and facial block were administered. The surgical steps of MSICS have been described previously. ${ }^{4}$ Briefly, this involved a fornix-based conjunctival flap, a partial thickness $6.5-7.5 \mathrm{~mm}$ frown-shaped scleral incision, and a scleral tunnel, which extended into clear cornea. Trypan blue was used to stain the anterior capsule. A continuous curvilinear capsulorhexis (CCC) of 5-6 mm was performed, followed by hydrodissection. A Sinskey hook was used to extract one pole of the nucleus outside the capsular bag, the rest of the nucleus was wheeled out into anterior chamber and
${ }^{1}$ Cataract Microsurgery Service, Aravind Eye Hospital, Pondicherry, India

${ }^{2}$ Department of Ophthalmology, The Eye Institute at Tan Tock Seng Hospital, National Healthcare Group, Singapore

Correspondence: $\mathrm{R}$ Venkatesh, Aravind Eye Hospital, Thavalakuppam, Pondicherry 605 007, India

Tel: + 9104132619100 Fax: 9104132618848

E-mail: venkatesh@ pondy.aravind.org

Received: 28 August 2007 Accepted in revised form: 20 May 2008

Published online: 20 June 2008

The authors have not received any financial support in the preparation of this manuscript. Conflict of interest: None 
extracted out of the eye using an irrigating vectis, After aspirating the cortex, a $6 \mathrm{~mm}$ optic PMMA posterior chamber intraocular lens was implanted.

Table 1 Complications on the first postoperative day using the OCTET classification

\begin{tabular}{llc}
\hline Complication & $\begin{array}{l}\text { OCTET } \\
\text { grading }\end{array}$ & $\begin{array}{c}\text { Number } \\
(\%) \\
\mathrm{n}=102\end{array}$ \\
\hline Corneal oedema with DM folds $>10$ & I & $15(14.7)$ \\
Corneal oedema with DM folds $<10$ & I & $5(4.9)$ \\
Mild iritis & I & $6(5.9)$ \\
Moderate iritis with fibrin membrane & II & $3(2.9)$ \\
Overall & - & $29(28.4)$ \\
\hline
\end{tabular}

$\mathrm{DM}=$ descemet's membrane; OCTET $=$ Oxford Cataract Treatment and Evaluation Team.

Table 2 40th postoperative day uncorrected and best-corrected visual acuity

\begin{tabular}{lcc}
\hline & Postoperative UCVA & Postoperative BCVA \\
\hline Better than 6/18 & $80(78.4 \%)$ & $99(97.1 \%)$ \\
6/18-6/60 & $22(21.5 \%)$ & $3(2.9 \%)$ \\
Worse than 6/60 & 0 & 0 \\
\hline
\end{tabular}

$\mathrm{BCVA}=$ best-corrected visual acuity; $\mathrm{UCVA}=$ uncorrected visual acuity.
The intraoperative and postoperative complications were graded according to Oxford Cataract Treatment and Evaluation Team (OCTET) classification. ${ }^{5}$ On the 40 th postoperative day, a complete ophthalmic examination was performed.

\section{Results}

The mean age of the 102 patients was 66.2 years (range, $38-85$ years, $\mathrm{SD} \pm 9.3)$. There were 41 male patients $(40.2 \%)$ and 61 female patients (59.8\%). The preoperative best-corrected visual acuity (BCVA) ranged from $6 / 24$ to light perception, with 79 patients $(77.5 \%)$ having BCVA worse than $6 / 60$.

Intraoperatively, superior scleral tunnel (SST) was constructed in 31 eyes (30.4\%) and temporal scleral tunnel (TST) in 71 eyes (69.6\%). Two patients had posterior capsule rupture, with anterior vitrectomy and implantation of IOL in the sulcus. On the first postoperative day, a total of 29 complications were noted in 23 eyes according to the OCTET classification (Table 1).

The visual outcomes are shown in Table 2. On the 40th postoperative day, 99 patients (97.1\%) achieved BCVA of $6 / 18$ or better. Of the remaining three patients, two had diabetic macular oedema and one had cystoid macular oedema. No patients had visual acuity worse than 6/60.

Table 3 Comparison of patients with SST $v$ S TST

\begin{tabular}{|c|c|c|c|}
\hline & $\begin{array}{c}S S T \\
\mathrm{~N}=31\end{array}$ & $\begin{array}{c}T S T \\
\mathrm{~N}=71\end{array}$ & P-value \\
\hline \multicolumn{4}{|l|}{ Age } \\
\hline Mean $( \pm S D)$, years & $64.9(10.0)$ & $66.8(9.0)$ & 0.363 \\
\hline \multicolumn{4}{|l|}{ Gender } \\
\hline Male (\%) & $11(35.5)$ & $30(42.3)$ & 0.661 \\
\hline Female $(\%)$ & $20(64.5)$ & $41(57.7)$ & \\
\hline \multicolumn{4}{|l|}{ Operated eye } \\
\hline Right (\%) & $21(67.7)$ & $35(49.3)$ & 0.129 \\
\hline Left $(\%)$ & $10(32.3)$ & $36(50.7)$ & \\
\hline \multicolumn{4}{|l|}{ Postoperative astigmatism } \\
\hline Mean $( \pm S D), D$ & $-1.08( \pm 0.74)$ & $-0.72( \pm 0.66)$ & $0.017^{*}$ \\
\hline Median (range) & $-1.00(-3.00-0.00)$ & $-0.75(-2.50-0.00)$ & \\
\hline \multicolumn{4}{|l|}{ Postoperative UCVA } \\
\hline Better than $6 / 18$ & $17(54.8)$ & $48(67.6)$ & 0.256 \\
\hline $6 / 18-6 / 60$ & $14(45.2)$ & $23(32.4)$ & \\
\hline Worse than $6 / 60$ & 0 & 0 & \\
\hline \multicolumn{4}{|l|}{ Postoperative BCVA } \\
\hline Better than $6 / 18$ & $30(96.8)$ & $67(94.4)$ & 1.000 \\
\hline $6 / 18-6 / 60$ & $1(3.2)$ & $4(5.6)$ & \\
\hline Worse than $6 / 60$ & 0 & 0 & \\
\hline
\end{tabular}

$\mathrm{BCVA}=$ best-corrected visual acuity; SST = superior scleral tunnel; $\mathrm{TST}=$ temporal scleral tunnel; UCVA = uncorrected visual acuity. *Statistically significant at the $5 \%$ level. 
Patients in the SST group had significantly higher postoperative astigmatism compared to the TST group (-1.08D vs $-0.72 \mathrm{D}, P=0.017$; Table 3 ). There were more patients with UCVA better than $6 / 18$ in the TST group $(67.6 \%)$ compared to the SST group (54.8\%). However, there were no significant differences in the complication rates, corneal oedema, inflammation, or BCVA between the two groups.

\section{Discussion}

Our study demonstrates that MSICS achieved good visual outcomes in $97.1 \%$ of patients with low complication rates. Performing phacoemulsification on brunescent cataracts involves the risk of endothelial damage, zonulysis, posterior capsule rupture, and postoperative corneal oedema. ${ }^{6,7}$ In a study on phacoemulsification in patients with $\mathrm{BBC}^{3} 28.1 \%$ of the patients developing corneal oedema in contrast to $19.6 \%$ of patients in our study. In that study, 13\% developed a wound-site thermal injury, whereas none occurred in our patients.

The safety of MSICS in BBC is further enhanced by the adjunctive use of Trypan blue dye before performing the CCC. The dye-stained capsular rim is distinctly visible throughout the surgery and any compromise to the capsular bag can be easily detected and relaxing incisions made to prevent intracapsular removal of nucleus. ${ }^{8,9}$

By the 40th postoperative day, UCVA and BCVA of better than 6/18 (the WHO criteria for good visual outcome) was achieved in 78.4 and $97.1 \%$ of our patients, respectively. The visual results are similar to randomised control trails comparing phacoemulsification and MSICS performed in India and Nepal. ${ }^{10,11}$

BBC cataracts may require a larger scleral tunnel (6.5$7.5 \mathrm{~mm}$ ) for extraction of nucleus from the eye, which can induce postoperative corneal astigmatism. A temporal incision may help control corneal astigmatism. In our study, the TST group had lower astigmatism than the SST group $(-1.08 \mathrm{D}$ vs $-0.72 \mathrm{D})$. A higher proportion of patients in the TST group had UCVA of $6 / 18$ or better $(67.6 \%)$ compared to the SST group (54.8\%).
In developing countries, many patients have BBC. Our study demonstrates that MSICS is a safe and effective treatment for patients with BBC.

\section{References}

1 Gogate PM, Deshpande M, Wormald RP, Deshpande R, Kulkarni SR. Extracapsular cataract surgery compared with manual small incision cataract surgery in community eye care setting in western India: a randomised controlled trial. Br J Ophthalmol 2003; 87: 667-672.

2 Muralikrishnan R, Venkatesh R, Prajna NV, Frick KD. Economic cost of cataract surgery procedures in an established eye care centre in Southern India. Ophthalmic Epidemiol 2004; 11: 369-380.

3 Singh R, Vasavada A, Janaswamy G. Phacoemulsification of brunescent and black cataracts. J Cataract Refract Surg 2001; 27: 1762-1769.

4 Venkatesh R, Muralikrishnan R, Balent LC, Prakash SK, Prajna NV. Outcomes of high volume cataract surgeries in a developing country. Br J Ophthalmol 2005; 89: 1079-1083.

5 Oxford Cataract Treatment and Evaluation Team. Use of grading system in evaluation of complications in a randomized controlled trial. Br J Ophthalmol 1986; 70: 411-414.

6 Hayashi K, Hayashi H, Nakao F. Risk factors for corneal endothelial injury during phacoemulsification. J Cataract Refract Surg 1996; 22: 1079-1084 23.

7 Hayashi K, Nakao F, Hayashi F. Corneal endothelial loss after phacoemulsification using nucleus cracking procedures. J Cataract Refract Surg 1994; 20: 44-47.

8 Venkatesh R, Das MR, Prashanth S, Muralikrishnan R. Manual small incision cataract surgery in white cataracts. Indian J Ophthalmol 2005; 53: 181-184.

9 Venkatesh R, Tan CSH, Kumar TT, Ravindran RD. Safety and efficacy of manual small incision cataract surgery for phacolytic glaucoma. Br J Ophthalmol 2007; 91: 279-281.

10 Ruit S, Tabin G, Chang D, Bajracharya L, Kline DC, Richheimer $\mathrm{W}$ et al. A prospective randomized clinical trial of phacoemulsification vs manual sutureless small-incision extracapsular cataract surgery in Nepal. Am J Ophthalmol 2007; 143: 32-38.

11 Gogate PM, Kulkarni SR, Krishnaiah S, Deshpande RD, Joshi SA, Palimker A et al. Safety and efficacy of phacoemulsification compared with manual small incision cataract surgery by a randomized controlled clinical trial. Ophthalmology 2005; 112: 869-874. 